\title{
Editorial
}

\section{Analysis and Models in Interdisciplinary Mathematics 2016}

\author{
J. C. Cortés, ${ }^{1}$ B. Chen-Charpentier, ${ }^{2}$ R. Company, ${ }^{1}$ Francisco J. Solis, ${ }^{3}$ \\ J. R. Torregrosa, ${ }^{1}$ and R. J. Villanueva ${ }^{1}$ \\ ${ }^{1}$ Instituto Universitario de Matemática Multidisciplinar, Universitat Politècnica de València, Camino de Vera, s/n, \\ 46022 Valencia, Spain \\ ${ }^{2}$ Department of Mathematics, University of Texas at Arlington, Arlington, TX 76019-0408, USA \\ ${ }^{3}$ CIMAT, Jalisco, s/n, 36240 Col. Valenciana, GTO, Mexico
}

Correspondence should be addressed to J. C. Cortés; jccortes@mat.upv.es

Received 23 November 2016; Accepted 23 November 2016

Copyright (c) 2016 J. C. Cortés et al. This is an open access article distributed under the Creative Commons Attribution License, which permits unrestricted use, distribution, and reproduction in any medium, provided the original work is properly cited.

According to the traditional course of action of this journal, this special issue includes a wide range of topics related to mathematical modelling, paying particular attention to social sciences, economics, finance, and uncertainty quantification. The aim of this issue is to provide a selected number of contributed papers that cover the area of mathematical modelling, giving a rather fair picture of the current research interests in this scientific field. It is expected that all the manuscripts comprised in this piece can contribute to enlarging both the foundations and applications of mathematical modeling.

This special issue has the vocation of continuing the previous ones entitled "Analysis and Models in Interdisciplinary Mathematics" and "Analysis and Models in Interdisciplinary Mathematics 2015" published in this journal in 2014 and 2015, respectively. The accepted papers have been selected after reviewing in order to integrate novel mathematical models and methods not only in the scope of traditional natural sciences but also in opening the scope to the social sciences framework. Also, theory and data-driven models, even in a synergy that gives rise to producing fertile, multidisciplinary, and hybrid models, have been considered.

Traditionally, mathematical modelling has been understood just as part of applied mathematics. This could be justified because, at its beginning, engineers were the main practitioners of this area of mathematics, developing mathematical models for solving engineering problems in natural sciences. However, this special issue also pursues providing mathematical modeling in contexts as social behavior, economics, and finance. Analysis methods and models in social sciences are similar to those of natural sciences including engineering, with the only difference that instead of using principles of nature one uses principles or theories from experts of such sciences. The special issue has also been dedicated to provide a window for the scientific community where mathematical models that account for uncertainty quantification within the framework of random differential equations and modelling can be shown.

It is our professional hope that the papers published in this volume can inspire and help other colleagues to some extent.

\section{Acknowledgments}

We thank all authors, participants, sponsors, editorial team, anonymous referees, and organizing team for their contribution in this special issue.

\author{
J. C. Cortés \\ B. Chen-Charpentier \\ R. Company \\ Francisco J. Solis \\ J. R. Torregrosa \\ R. J. Villanueva
}




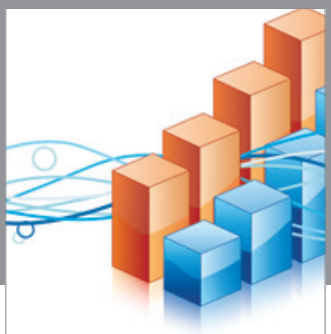

Advances in

Operations Research

vatem alat4

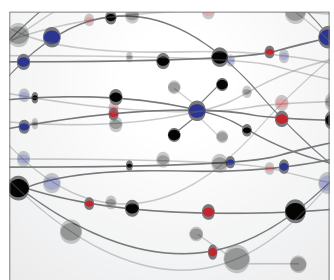

\section{The Scientific} World Journal
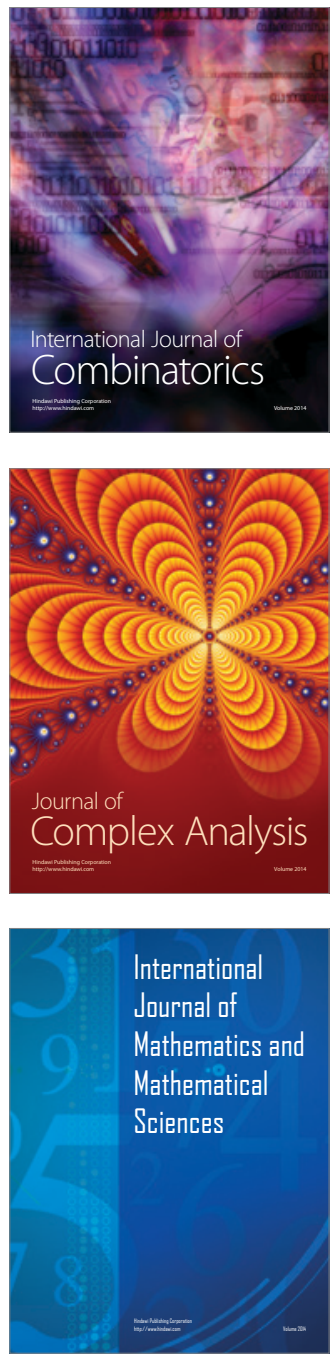
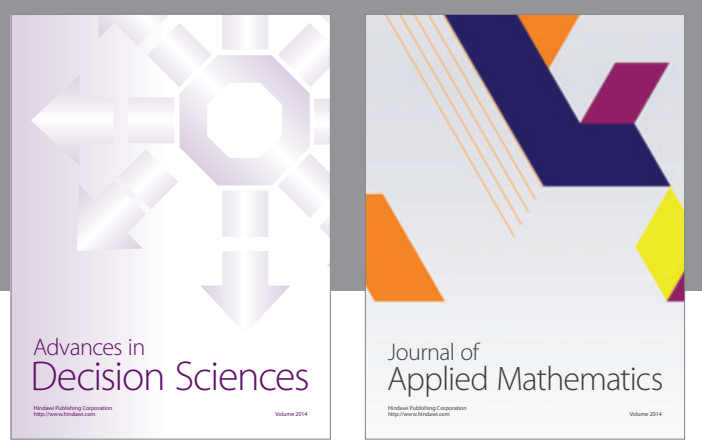

Algebra

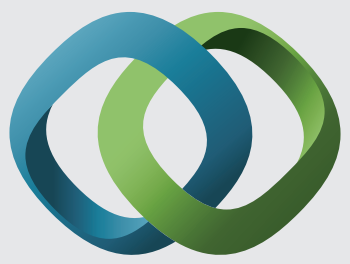

\section{Hindawi}

Submit your manuscripts at

http://www.hindawi.com
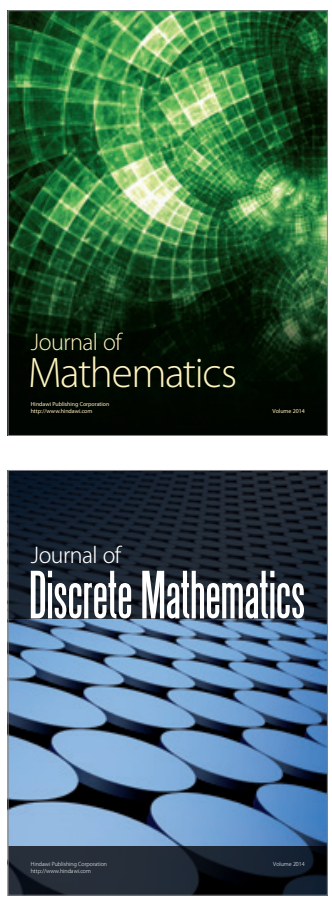

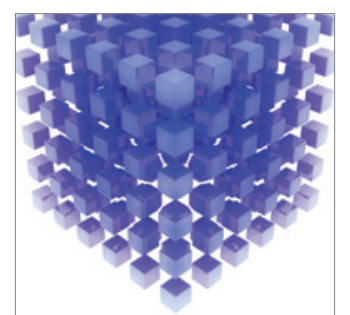

Mathematical Problems in Engineering
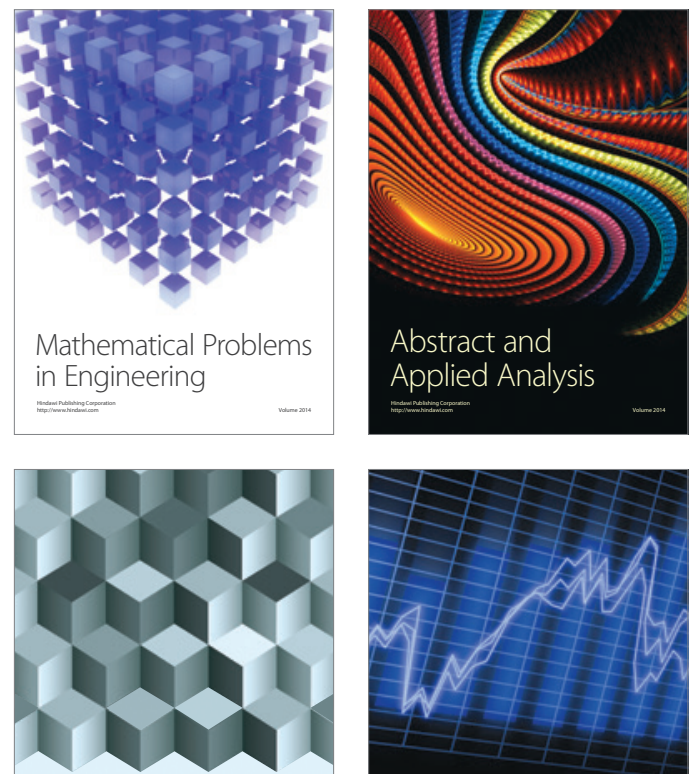

Journal of

Function Spaces

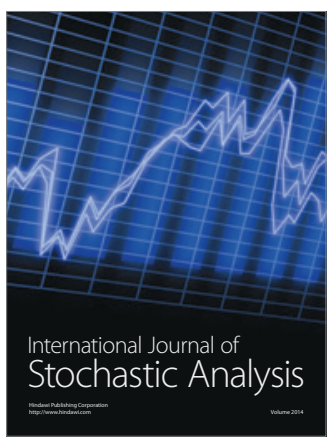

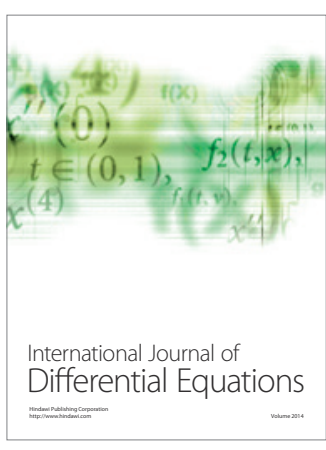
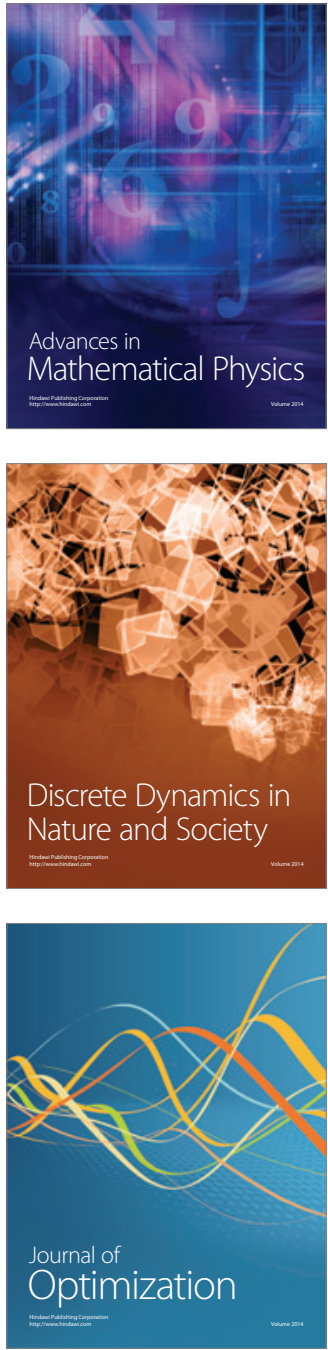